\title{
Relaciones Anatómicas del Cuerpo Adiposo de la Mejilla Aso- ciadas a Complicaciones de bichectomía. A Propósito de un Caso
}

\author{
Anatomical Relationships of the Adipose Buccal Fat Pad \\ Associated with Bichectomy Complications. About a Case
}

Oscar Hernández ${ }^{1}$; Jorge Altamirano ${ }^{2}$; Reinaldo Soto ${ }^{1,3}$ \& Alejandro Rivera ${ }^{2,3}$

HERNÁNDEZ, O.; ALTAMIRANO, J.; SOTO, R. \& RIVERA, A. Relaciones anatómicas del cuerpo adiposo de la mejilla asociadas a complicaciones de bichectomía. A propósito de un caso. Int. J. Morphol., 39(1):123-133, 2021.

RESUMEN: El presente estudio busca entregar conocimientos y aspectos importantes de las estructuras anatómicas asociadas al cuerpo adiposo de la mejilla (CAM), a consecuencia del reporte de un caso de complicación posterior a una bichectomía. Conocer los elementos anatómicos y sus relaciones con el CAM es de vital importancia, ya que en la actualidad son muchos los cursos y pasadas que se realizan para odontólogos generales y especialistas para el retiro del CAM, esto producto de la creciente demanda estética de las personas por presentar un perfil más fino y estilizado. El caso presentado corresponde a una mujer que consultó al servicio de urgencia por aumento de volumen facial posterior a la extracción del CAM, el cual se diagnosticó como sialocele, consecutivo a daño del conducto parotídeo. Al revisar la literatura y observar en nuestros preparados anatómicos se aprecia la cercanía de elementos de importancia, como son el conducto parotídeo, los ramos cigomático y bucal del nervio facial, vasos faciales como la arteria facial, vena facial y arteria transversa facial, es por esto que es necesario conocer en detalle la zona a intervenir y considerar las posibles variaciones anatómicas de estos elementos, para así evitar dañarlos durante el procedimiento, tomando una actitud preventiva. Apuntado entonces al objetivo de este estudio, fue lograr entregar información anatómica precisa y concisa de los elementos de importancia próximos o inmersos en el CAM, para prevenir la iatrogenia de estos.

PALABRAS CLAVE: Anatomía; Tejido graso / cirugía; Mejilla /cirugía, estética; métodos / lipectomia.

\section{INTRODUCCIÓN}

Bichectomía es el nombre que se le da al procedimiento quirúrgico que tiene como objetivo la resección del cuerpo adiposo de la mejilla (CAM), eliminando la porción correspondiente a la almohadilla de grasa de "Bichat", el cual incluye alrededor del 30 a $40 \%$ de esta estructura (Stuzin et al., 1990). Este procedimiento entrega como resultado la reducción del volumen en el tercio medio de la cara.

Heister (1732) fue el primer autor en describir el CAM como una estructura glandular. Sin embargo, recién en 1802, Marie-Francois Xavier Bichat describió esta estructura anatómica como un tejido adiposo, llamado "Bichat Ball”, y su aplicación clínica se produce en las áreas médica y odontológica (Shoja et al., 2008).

El CAM es una estructura de tejido graso envuelto por una fina capa de tejido conectivo, la cual presenta forma piramidal, extendiéndose en masa en la región geniana, tem- poral e infratemporal, y en profundidad hasta la región pterigopalatina, comunicándose en algunos casos con la órbita, a través de la fisura orbitaria inferior. Esta estructura anatómica llena el espacio masticatorio, separando los músculos masticadores entre sí (Zhang et al., 2002). Tiene una función mecánica, facilitadora de los movimientos masticatorios, producto de estar cubierta por una delgada cápsula fibrosa, aislándola del contacto directo con los tejidos circundantes, lo que permite los movimientos de succión en los recién nacidos y la masticación en etapas posteriores. Por lo tanto, el CAM puede considerarse una estructura deslizante que ayuda a movimientos de masticación y dearticulación de la palabra. Además, tiene una función estética, responsable de dar el contorno facial (Stuzin et al.; Zhang et al.). El CAM se encuentra en un área de la cara rodeada de estructuras anatómicas, como son las venas facial y facial profunda, vasos y nervios infraorbitarios y bucales, nervio facial y conducto parotídeo.

${ }^{1}$ Departamento de Morfología, Facultades de Odontología y Medicina, Universidad de los Andes, Santiago, Chile.

${ }^{2}$ Urgencia Dental y Maxilofacial, Unidad de Emergencias Adultos, Hospital Dr. Sotero del Rio, Santiago, Chile.

${ }^{3}$ Asignatura de Cirugía, Facultad de Odontología, Universidad de los Andes, Santiago, Chile. 
El procedimiento para reducir el CAM está indicado para personas que presenten alguna alteración estética del tercio medio de la cara, principalmente aquellos con mayor amplitud en sentido transversal, dándoles una redondez facial excesiva, con pesadez y aspecto no armónico. De esta manera, la escisión del CAM puede mejorar el contorno facial del paciente, proporcionando un adelgazamiento en las mejillas, logrando un contorno facial más suave y con líneas más simétricas, en las que un contorno facial cuadrado puede volverse cóncavo, y en consecuencia más delicado y armonioso (Dias et al., 2018; Moreira Júnior et al., 2018; Klüppel et al., 2018).

Esta técnica está indicada para fines funcionales y principalmente estéticos y se ha extendido bastante entre los profesionales del área de la salud, especialmente entre odontólogos, convirtiéndose una tendencia especialmente entre las mujeres. Con el creciente número de intervencio- nes quirúrgicas que involucran esta estructura, es necesario un conocimiento anatómico preciso de esta región para evitar la iatrogenia de estas estructuras, que puede causar secuelas temporales y permanentes. Sin embargo, hoy en día existen pocos artículos de sus complicaciones y alteraciones en estructuras anatómicas relacionadas con el CAM, las que pueden generar múltiples alteraciones tanto funcionales como estéticas.

Desarrollo. El cuerpo adiposo de la mejilla (CAM), es una estructura de tejido adiposo simple alojado en espacios superficiales y profundos de la cara, el cual, según descripción clásica, presenta un cuerpo central, con cuatro prolongaciones; bucal, pterigoidea, pterigopalatina y temporal, ubicadas entre los músculos masticadores (Stuzin et al.; Matarasso, 1991; Tostevin \& Ellis, 1995; Kahn et al., 2000). Otra descripción más actual es en base a lobos, siendo estos 3: anterior, intermedio y posterior, esta descripción en torno

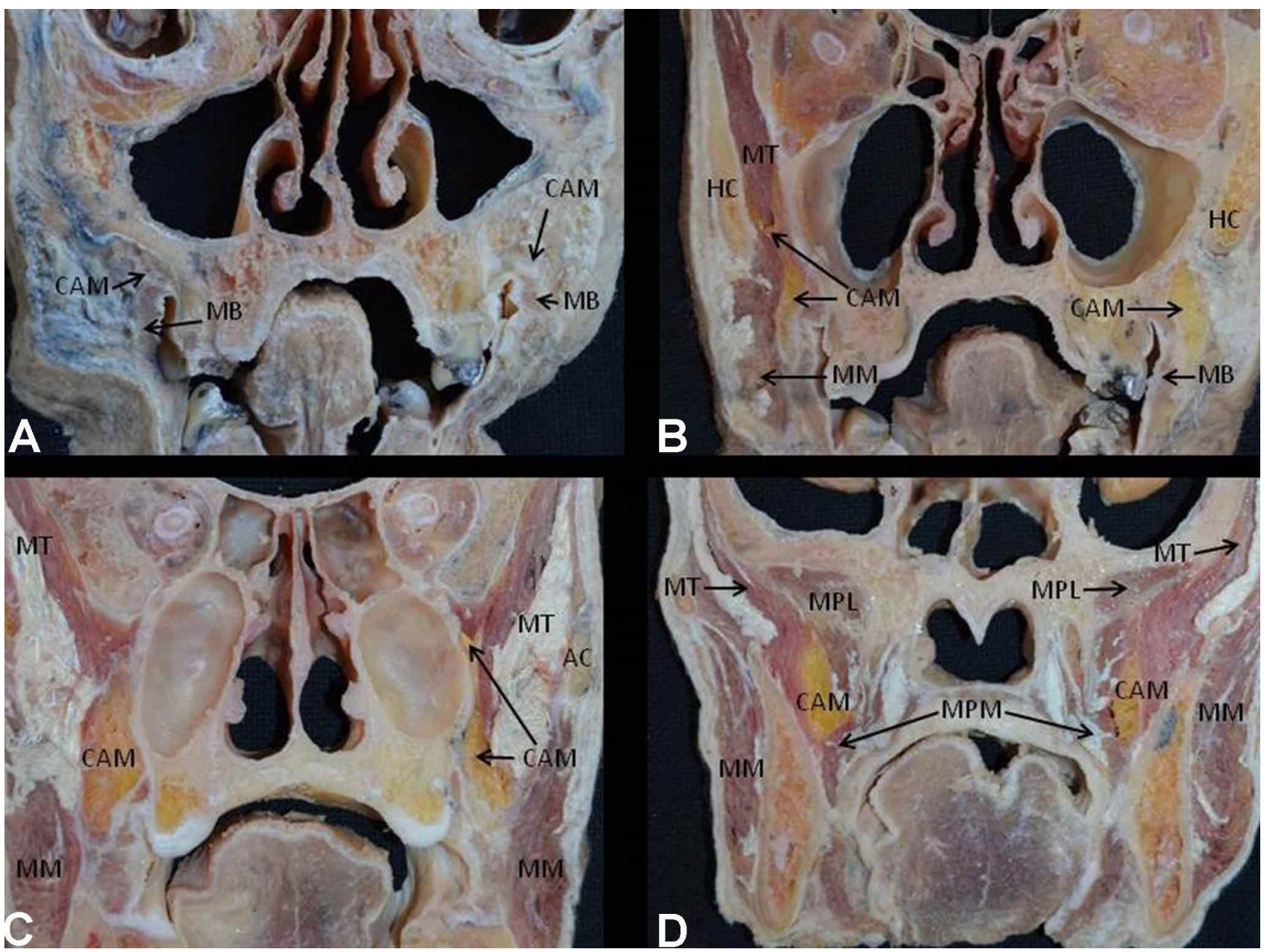

Fig. 1. Visión anterior cortes coronales de cara. A: corte a la altura del $2^{\circ}$ premolar superior. B: corte a 1,5 cm. posterior de A. C: corte a $1 \mathrm{~cm}$. posterior de B. D: corte a 1,5 cm posterior de C. CAM: cuerpo adiposo de la mejilla; MB: músculo buccinador; MT: músculo temporal; MM: músculo masetero; MPL: músculo pterigoideo lateral; MPM: músculo pterigoideo medial: HC: hueso cigomático; AC: arco cigomático. 
a lobos se basa en los ligamentos que los fijan, la irrigación y el encapsulamiento que estos presentan (Zhang et al.; Arce, 2007). A grandes rasgos el CAM, se encuentra limitado por el músculo masetero, el tendón del músculo temporal y el músculo pterigoideo medial en la parte posterior, el músculo buccinador y su fascia por medial, el primer plano de los músculos faciales por lateral y el conducto parotídeo junto a su delgada lámina fascial por anterior (Matarasso; AltschillerMardones et al., 2018).

El volumen total del CAM es variable, algunos autores lo definen en un promedio de 9,6 ml (Hassani et al., 2016; Klüppel et al.), otros en $10 \mathrm{ml}$ (Tostevin \& Ellis). Además, el componente graso que conforma esta estructura permanece relativamente estable en volumen durante toda la vida debido a que, histológicamente, el CAM está compuesto por el mismo tipo de tejido adiposo que se encuentra en otras partes del cuerpo. Sin embargo, no es consumido por la pérdida de peso, un proceso que si ocurre con la grasa de otras zonas (Tostevin \& Ellis; Hassani et al.; Klüppel et al.) (Fig. 1).

Ligamentos de fijación. Los elementos de fijación del cuerpo adiposo de la mejilla son ligamentos, que unen los diferentes lobos a estructuras anatómicas cercanas para mantenerlo en posición. Se indica que cada lobo tiene entre 2 a 4 ligamentos que también favorecen la entrada de los diferentes vasos sanguíneos que nutren el CAM. Los ligamentos han sido resumidos en la Tabla I (Zhang et al.; Arce).

Irrigación: La irrigación del CAM,está dado por tres grandes vasos, los cuales conforman un rico plexo arterial en la profundidad, las arterías involucradas en la formación de este plexo son la maxilar, con sus ramas temporales profundas, bucal y alveolar posterior superior, la arteria transversa facial colateral de la arteria temporal superficial y ramas directas de la arteria facial (Stuzin et al.; Tostevin \& Ellis; Zhang et al.; Hassani et al.; Altschiller-Mardones et al.). El drenaje venoso se da vía vena facial, principalmente y plexo pterigoalveolar (Tostevin \& Ellis; Hassani et al.). La arteria $\mathrm{y}$ vena facial ascienden en el mismo plano que el CAM, y marcan la extensión anterior en la mejilla del CAM. La arteria transversa facial trascurre por superior al CAM y al conducto parotídeo. La arteria temporal media entrega ramas para la prolongación temporal superficial (Stuzin et al.). La arteria maxilar y facial, proveen irrigación a los lobos anterior, intermedio y posterior. Zhang et al. señalaron que cada lobo presenta su irrigación definida, la cual sirve para determinar y describir su clasificación según lobos. En la Tabla II se muestra resumen de la irrigación según Zhang et al.

Lobos: El lobo anterior se ubica por inferior al hueso cigomático, extendiéndose anteriormente al buccinador, maxilar y el espacio profundo comprendido en el cuadrilátero formado por el músculo elevador del labio superior por anterior y el músculo cigomático mayor por posterior, músculo orbicular del ojo por superior y músculo orbicular oral por inferior. La forma que presenta es triangular, se compone de múltiples tabiques de tejido conectivo que separan el tejido graso en masas más pequeñas (Zhang et al.). Se comunica con el lobo intermedio y posterior mediante tejido conectivo laxo y con la membrana que cubre al buccinador mediante tejido conectivo denso alrededor de la apertura del conducto parotídeo, junto con el lobo posterior (Zhang et al.).

El lobo intermedio se encuentra en el espacio comprendido entre el lobo posterior, por lateral al maxilar y el lobo anterior. Si bien existe una separación entre el lobo intermedio y posterior en sus porciones superiores por un tabique membranoso, en la porción inferior no se encuentra tal división (Stuzin et al.; Zhang et al.).

Tabla I. Resumen de ligamentos de fijación del cuerpo adiposo de la mejilla.

\begin{tabular}{lll}
\hline Ligamento & Origen & Inserción \\
\hline Ligamento maxilar (condensación fibrosa) & Lobo anterior & Maxilar \\
Ligamento cigomático posterior & Lobo intermedio & Proceso cigomático \\
Ligamentos infraorbitario medial y lateral & Lobo intermedio (lados medial y lateral) & Borde infraorbitario \\
Ligamento tendón temporal & Lobo posterior & Posterior al tendón del temporal \\
Ligamento buccinador & Lobo anterior & Membrana buccinatriz. \\
\hline
\end{tabular}

Tabla II. Resumen de la irrigación del cuerpo adiposo de la mejilla, descrita en base a lobos.

\begin{tabular}{llll}
\hline & & Arteria maxilar & \multicolumn{1}{c}{ Arteria facial } \\
\hline Lobo anterior & Porción superior & Alveolar posterior superior & Rama anterior de la ar \\
& Porción inferior & Arteria bucal & bucal inferior \\
Lobo intermedio & Porción superior & Alveolar posterior superior & Rama anterior de la ar \\
& Porción distal e inferior & Arteria bucal & bucal inferior \\
Lobo posterior & Porción superior & Arteria bucal & Rama posterior de la \\
& Porción media & Arterias del músculo pterigoideo. & arteria bucal inferior \\
\hline
\end{tabular}


El lobo posterior queda comprendido en los espacios profundos de la cara. Se extiende hasta la fisura orbitaria inferior y los espacios que rodean el músculo temporal, hasta el margen superior del cuerpo mandibular, y de regreso al margen anterior del tendón temporal y la rama. Del lobo posterior se desprenden las prolongaciones bucal, pterigopalatina, pterigoidea y temporal (Zhang et al.; Arce). La prolongación bucal es la porción inferior del lobo posterior, por inferior del conducto parotídeo (Zhang et al.), sobrepasa anteriormente al margen anterior del masetero, donde se hace superficial, dándole el contorno facial a la mejilla. Desde aquí se extiende profundo al conducto parotídeo, entre los músculos masetero y buccinador, y descansa sobre la fascia buccinatriz, que cubre la superficie externa del buccinador. El límite anterior se encuentra justo posterior al sitio donde el conducto parotídeo perfora el buccinador (Tostevin \& Ellis; Zhang et al.) (Fig. 2).

Relaciones anatómicas: En su proximidad el CAM presenta relaciones de importancia como son el nervio facial, el conducto parotídeo, vena facial, los nervios y vasos infraorbitarios y bucales, arteria facial y facial transversa.
Pudiendo distribuirse superficial o por el espesor del cuerpo adiposo de la mejilla (Zhang et al.; Hassani et al.; Benjamin \& Reish, 2018). Los más importantes desde un punto de vista funcional son el conducto parotídeo y el nervio facial, con sus ramos cigomático y bucal, que cruzan las superficies anterior y lateral del CAM (Fig. 3).

El nervio facial, con sus ramos bucal y cigomático, tiene una estrecha relación con la prolongación bucal del CAM (Hwang et al., 2005; Hassani et al.). Específicamente su ramo bucal, el cual es inferior al conducto parotídeo en su trayecto a través de la región maseterina y geniana (Matarasso). El nervio bucal, ramo del nervio mandibular, proviene de la región infratemporal específicamente del espacio pterigomandibular y en su recorrido es acompañado de sus vasos. Tanto nervio como vasos están en una relación próxima o inmersos en el CAM (Stuzin et al.; Zhang et al.).

Se establece que el conducto parotídeo se extiende a lo largo de la superficie lateral de CAM, prolongación bucal, o perfora el lobo posterior antes de llegar a la superficie del músculo buccinador, pudiendo establecer tres formas de


Fig. 2. Visión inferior de cortes axiales de cabeza. El corte es levemente oblicuo hacia inferior e izquierda. A: corte a media altura de la rama mandibular. B: corte $1,5 \mathrm{~cm}$. superior de A. C: corte $1,5 \mathrm{~cm}$. superior a B. D: corte $1 \mathrm{~cm}$. superior a C. CAM: cuerpo adiposo de la mejilla; MB: músculo buccinador; AF: arteria facial; MT: músculo temporal; MM: músculo masetero; MPL: músculo pterigoideo lateral; MPM: músculo pterigoideo medial: HC: hueso cigomático; AC: arco cigomático; RM: rama mandibular; GP: glándula parótida; EPM: espacio pterigomandibular; EPF: espacio pterigofaríngeo. 

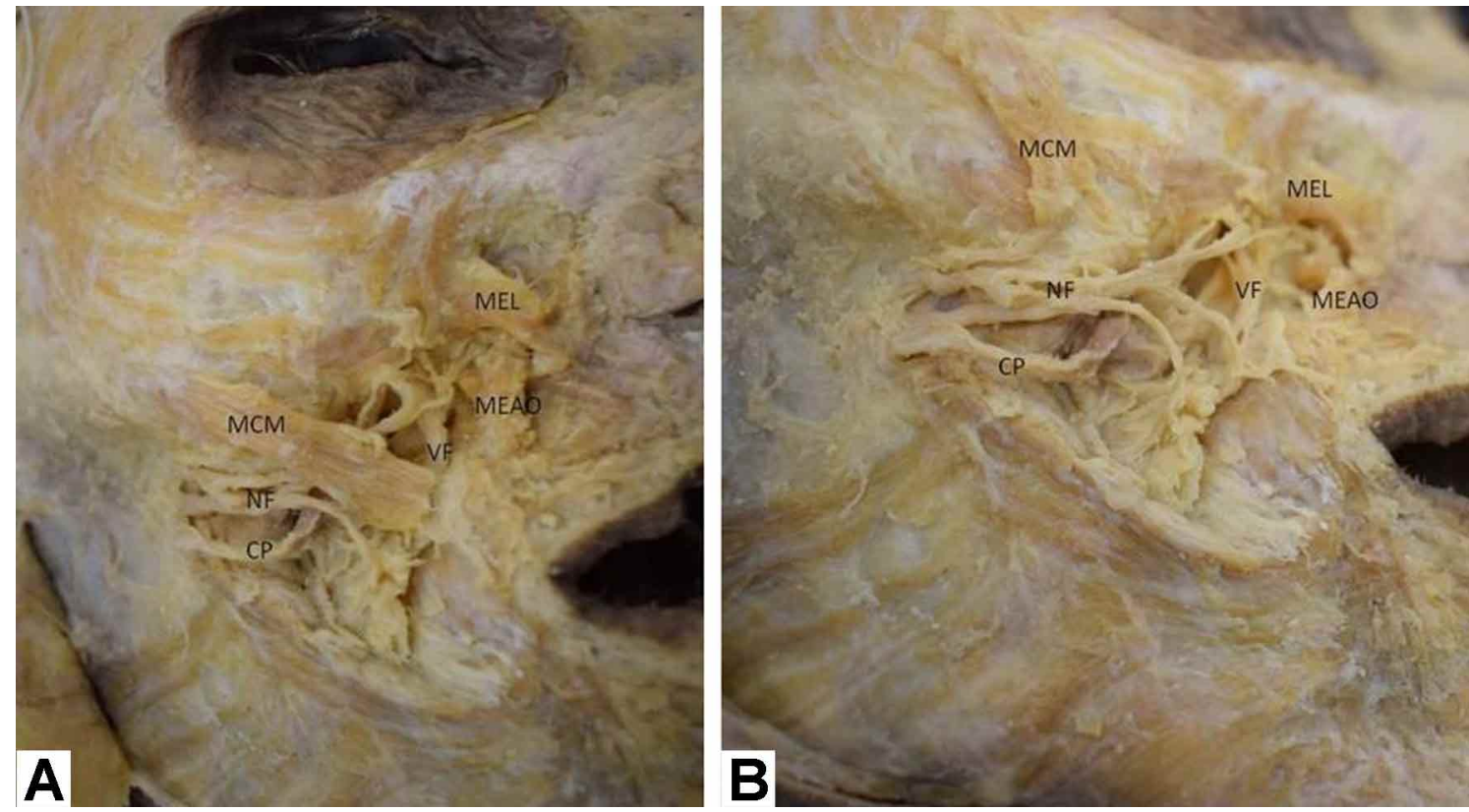

Fig. 3. Visión lateral derecha del plano superficial de la cara.A: MCM: músculo cigomático mayor; NF: nervio facial; DP: conducto parotídeo; VF: vena facial; MEAO: músculo elevador del ángulo oral; MEL: músculo elevador del labio.B: Músculo cigomático mayor rechazado hacia superior. Nervio facial y conducto parotídeo emergiendo de la celda parotídea.

relacionarse con ella. El conducto parotídeo puede estar superficial (42\%), por superior (36\%) o inmerso $(26 \%)$ en el CAM. (Hwang et al.; Hassani et al.), luego el conducto perfora el músculo buccinador y desemboca en la cavidad oral adyacente a los segundos molares superiores (Matarasso; Hwang et al.; Hassani et al.). El conducto parotídeo en su trayecto superficial y lateral a la prolongación bucal del CAM lo divide en una porción superior y otra inferior (Kahn et $a l$.), en la cual encontramos al nervio facial (Matarasso).

En la profundidad del CAM se establece un rico plexo vascular arterial que lo irriga. Las arterias que forman este plexo son las arterias temporales profundas, bucal y alveolar posterior superior, la arteria transversa facial y facial. Además, por proximidad, hacia anterior, el CAM en ocasiones establece una estrecha relación con la arteria infraorbitaria (Stuzin et al.; Tostevin \& Ellis; Zhang et al.; Hassani et al.; Altschiller-Mardones et al.). La arteria y vena facial ascienden en el mismo plano que el CAM, y marcan su extensión anterior en la mejilla. La arteria transversa facial trascurre por superior al CAM, y al conducto parotídeo. La arteria bucal, presenta el mismo trayecto que el nervio bucal (Stuzin et al.; Zhang et al.; Hassani et al.; Altschiller-Mardones et al.). La vena facial profunda, es un elemento vascular de importancia, ya que anastomosa el plexo pterigoalveolar con la vena facial, esto lo hace ingresando por el hiato buccinatomaseterino donde se relaciona con la prolongación bucal del CAM que emerge de la profundidad (Matarasso; Kahn et al.).
Técnica Quirúrgica. Existen dos abordajes para realizar la eliminación del cuerpo adiposo de la mejilla, uno intraoral y otro extraoral. La más utilizada y estudiada es mediante abordaje intraoral y se desarrolla bajo la infiltración de anestesia local, sin descartar la necesidad de anestesia general cuando el paciente requiere procedimientos quirúrgicos concomitantes. La incisión se puede realizar vertical hacia superior en relación con el primer y segundo molar superior de $2,5 \mathrm{~cm}$, a nivel de el surco gingivobucal como también horizontal a nivel de la línea alba de la mejilla (Jeannie H. Chung et al., 2018), respetando $1,5 \mathrm{~cm}$ posterior al agujero de salida del ducto parotídeo, teniendo como referencia el plano oclusal maxilar (Jinghong Xu y Yijia et al. 2013). Con instrumentos romos se divulsiona hasta observar la cápsula que recubre el cuerpo adiposo de la mejilla, esta debe perforarse para acceder, mientras es traccionado hacia anterior, medial e inferior, aplicando presión externa bajo el arco cigomático. Se utilizan pinzas kelly para tomar el CAM, manipulando a través de la incisión, traccionando y extrayendo suavemente, hasta que se corta, pretendiendo que este corte sea en el nivel del CAM que corresponde al arco cigomático. El muñón idealmente se coagula con un electrobisturí y la herida se empaqueta con una gasa empapada en solución de lidocaína y epinefrina, hasta comprobar que el tamaño y peso de ambos cuerpos adiposos extraídos sea similar. La herida se cierra con una sutura absorbible de calibre 3-0 a 5-0, con posterior comprobación de la permeabilidad del conducto parotídeo. Se coloca un vendaje de compresión elástica durante al menos de 3 días, apoyado 
de indicaciones farmacológicas que consideren antibioterapia, antiinflamatorios no esteroidales y esteroidales.

Complicaciones. Una serie de reportes de caso en 220 artículos encontrados en donde solo 8 cumplían con los criterios de inclusión para realizar una revisión sistemática de la literatura, mostró que en 71 pacientes sometidos al procedimiento de eliminación del cuerpo adiposo de la mejilla con o sin asociarse al procedimiento de estiramiento facial con técnica de ritidectomía, sólo el 8,45 \% (6 pacientes) presentaron complicaciones menores (Lucas-Borin Moura et al. 2018). Además, ningún estudio evaluó el envejecimiento facial y los efectos a largo plazo, por lo tanto, el efecto inofensivo del procedimiento para esas características no está claro.

Las complicaciones después de la extracción del cuerpo adiposo de la mejilla están muy relacionadas a noxa en las estructuras nobles adyacentes, e incluyen hematoma, infección, trismus, edema prolongado, lesión del nervio facial, lesión del conducto parotídeo, irregularidades y asimetrías del contorno facial. Durante las intervenciones para acceder intraoralmente al CAM, los hematomas pueden estar relacionados con un traumatismo en una rama buccinatriz inferior posterior de la arteria facial, que se desliza superficial al músculo buccinador y es la encargada de aportar la nutrición al lobo posterior del cuerpo adiposo de la mejilla (Klüppel et al.). Con respecto a los daños causados al conducto parotídeo, se relacionan por la proximidad de la incisión a éste y por no considerar las variaciones anatómicas del conducto, ya que puede presentarse superficial al lobo posterior (42\%), por superior $(36 \%)$ o inmerso $(26 \%)$ en esta (Hassani et al.).

\section{CASO CLÍNICO}

Paciente femenino de 26 años, fue derivado a nuestro centro asistencial, con diagnóstico de absceso geniano izquierdo, para evaluación de hospitalización y manejo quirúrgico. El paciente relató que 4 días antes de la consulta se había realizado un procedimiento de remoción del cuerpo adiposo de la mejilla bilateral, con dificultad intraoperatoria mayor en lado izquierdo, sin relato de complicaciones. Se le indicó amoxicilina $875 \mathrm{mg}$ con ácido clavulánico $125 \mathrm{mg}$ comprimidos, paracetamol $1 \mathrm{~g}$ e ibuprofeno $600 \mathrm{mg}$. El paciente presentó leve sangrado de herida quirúrgica de lado izquierdo hasta $36 \mathrm{~h}$ posterior al procedimiento y a las $48 \mathrm{~h}$ presentaba aumento de volumen de región geniana izquierda, de consistencia blanda, de límites definidos, dolor leve sin eritema ni cambios de temperatura de la piel relacionada.
A las 72 horas por indicación de su tratante inició terapia farmacológica con prednisona ( $20 \mathrm{mg}$ cada $8 \mathrm{~h}$ vía oral). Evolucionó con incremento en el aumento de volumen facial, expandiéndose a los espacios profundos y submandibular ipsilateral. Tuvo compromiso del estado físico general sin alteración del estado de conciencia, con adinamia, sensación febril no cuantificada, disfagia, odinofagia y limitación de apertura oral.

La peciente no presentaba antecedentes mórbidos relevantes, a excepción de una reconstrucción de clavícula derecha con injerto de cadera, controlada con tramadol y pregabalina a permanencia.

Al examen físico general presentó presión arterial de 160/96 mm/hg, pulso 101 ppm, eupnéica, buen estado nutritivo y sin alteración del estado de consciencia. Al examen facial presentó un aumento de volumen hemifacial izquierdo con compromiso de región geniana, submandibular y periorbitaria, con limitación de apertura palpebral. Presentaba eritema y consistencia firme en piel relacionada y limitación de apertura de la cavidad oral. Al examen intraoral se observaron heridas quirúrgicas en mucosa yugal bilateral en relación con primeros y segundo molares superiores, suturadas con Nylon. En lado izquierdo presentaba edema y eritema de tejido blando circundante, sin exudado, y con dolor a la exploración. El conducto parotídeo izquierdo no estaba permeable (Fig. 4).
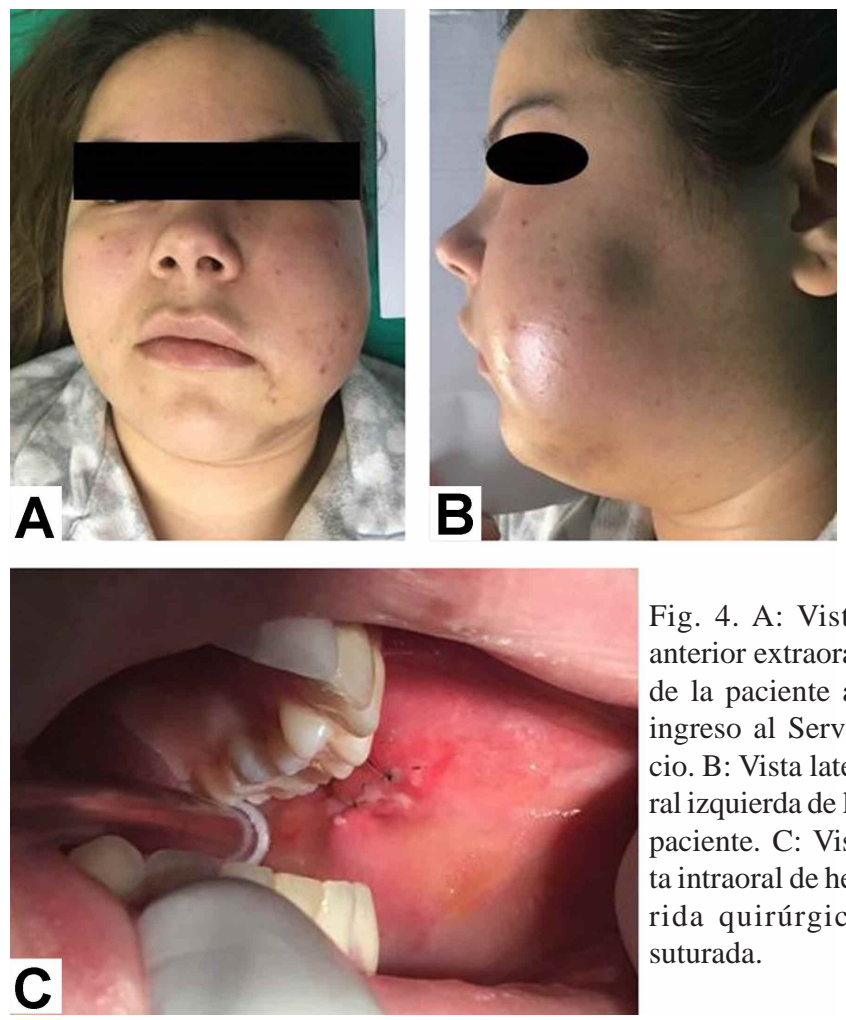

Fig. 4. A: Vista anterior extraoral de la paciente al ingreso al Servicio. B: Vista lateral izquierda de la paciente. C: Vista intraoral de herida quirúrgica suturada. 
Se solicitó un estudio imagenológico de tomografía axial computarizada de cabeza y cuello, donde se observó una gran área hipodensa de límites definidos en zona geniana izquierda, e inflamación del espacio subcutáneo (Figs. 5, 6 y 7).
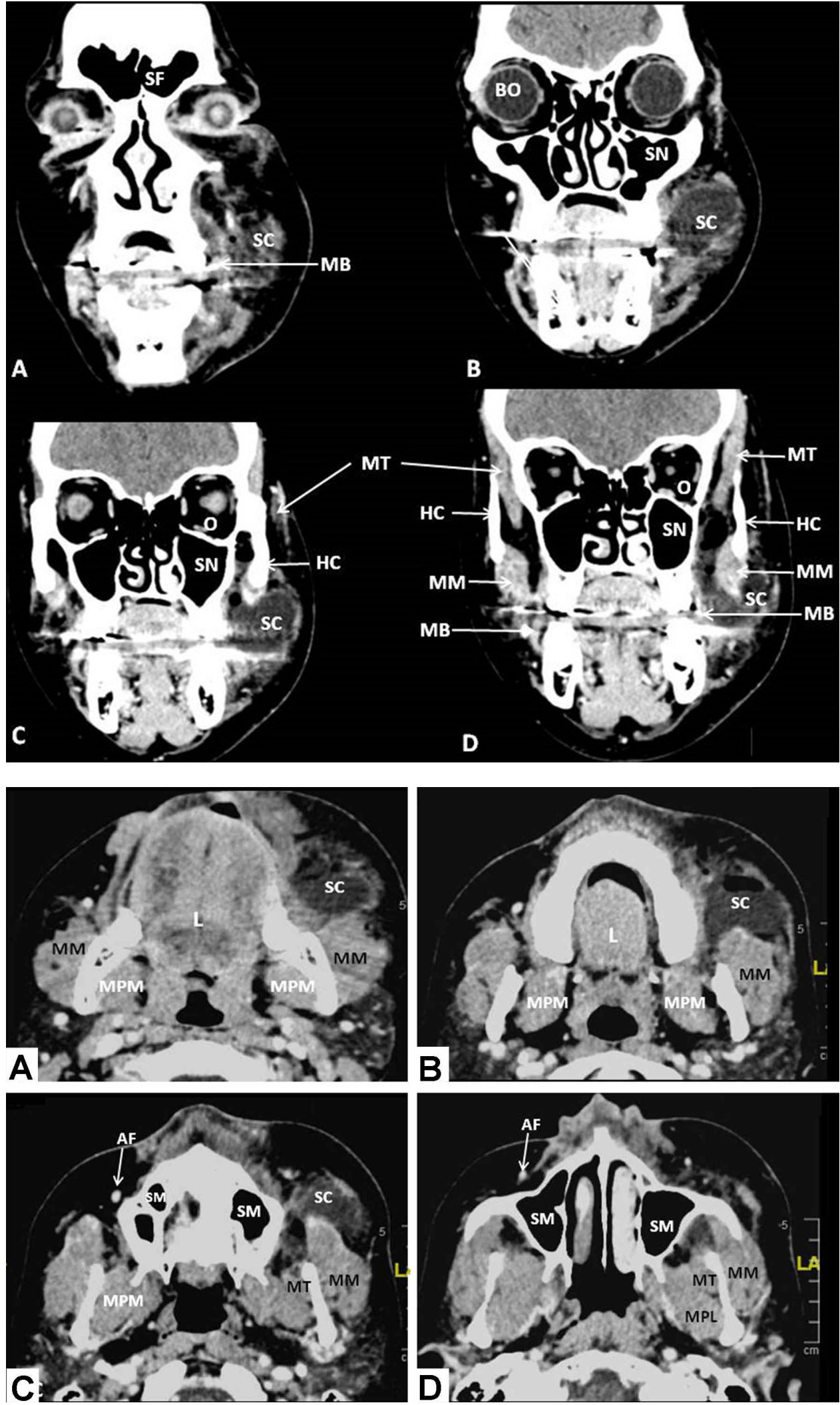
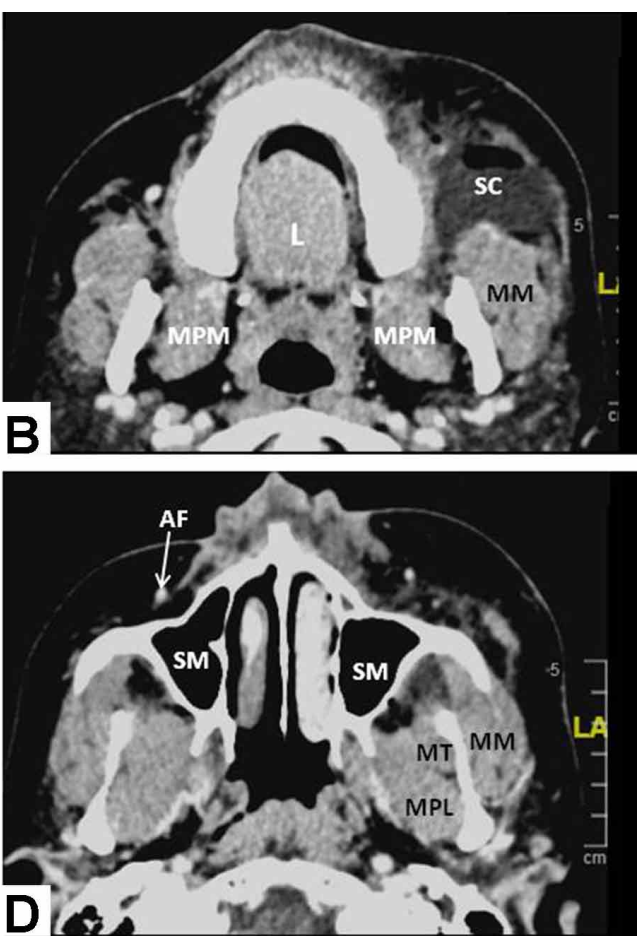

Fig. 5. Cortes coronales tomografía computada del caso. A: Corte coronal a nivel del primer premolar. B: $10 \mathrm{~mm}$ posterior de A. C: $10 \mathrm{~mm}$ posterior de B. D: $0,5 \mathrm{~mm}$ posterior de $\mathrm{C}$. $\mathrm{SF}$ : seno frontal; $\mathrm{BO}$ : bulbo ocular; SN: seno maxilar; O: órbita; SC: sialocele; HC: hueso cigomático; AC: arco cigomático; MT: músculo temporal; MM: músculo masetero; MB: músculo buccinador.

Fig. 6. Cortes axiales tomografía computada del caso. A: corte a media altura de la rama mandibular. B: $15 \mathrm{~mm}$ superior de A. C: 9 mm superior de B. D: 5 mm superior de C. SC: sialocele; SM: seno maxilar; L: lengua; MT: músculo temporal; MM: músculo masetero; MPL: músculo pterigoideo lateral; MPM: músculo pterigoideo medial; AF: arteria facial. HC: hueso cigomático; $\mathrm{AC}$ : arco cigomático. 

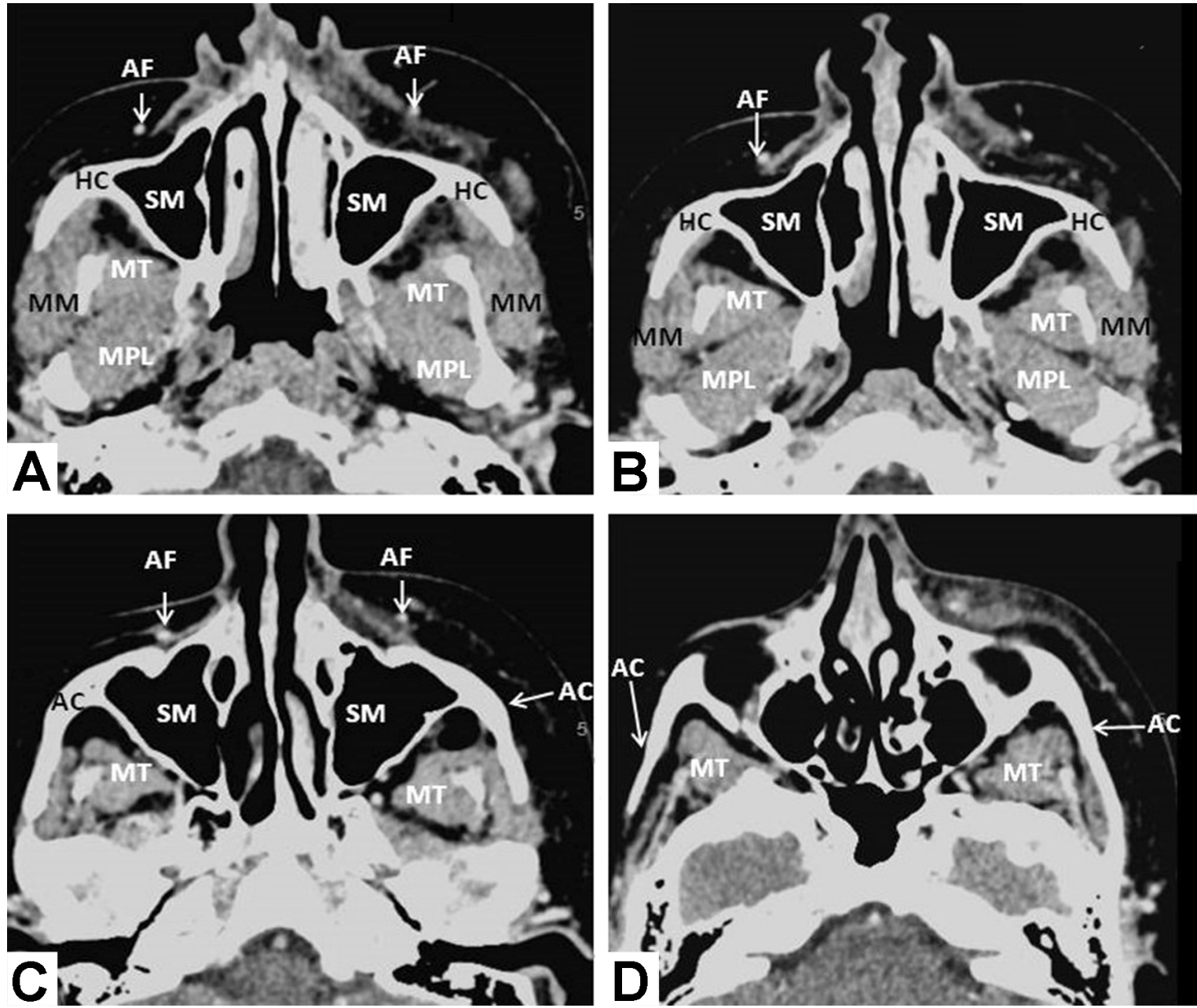

Fig. 7. Cortes axiales tomografía computada del caso. A: A la altura de la incisura mandibular. B: 3 mm superior de A. C: $7 \mathrm{~mm}$ superior de B. D: $8 \mathrm{~mm}$ superior a C. SC: sialocele; SM: seno maxilar; MT: músculo temporal; MM: músculo masetero; MPL: músculo pterigoideo lateral; MPM: músculo pterigoideo medial; AL: arteria facial. HC: hueso cigomático; AC: arco cigomático.

Diagnóstico: Sialocele en región geniana izquierda por obstrucción de conducto parotídeo.

Tratamiento. Bajo anestesia local de lidocaína al $2 \%$ con epinefrina 1:100.000, bloqueo nervio alveolar inferior y bucal izquierdo con refuerzo perifocal en herida quirúrgica de mucosa yugal, se realizó el retiro de sutura y una cuidadosa divulsión roma a través de la herida, obteniendo primero un vaciamiento de abundante exudado salival, para una vez disminuido el flujo, un exudado hemáticosalival. Se complementó y estimuló su salida con masaje infra cigomático y parotídeo. Paciente relató inmediato alivio de sintomatología aguda de dolor. (Fig. 8). Se lavó profusamente con suero fisiológico, no obstante no fue posible localizar el agujero de salida del conducto parotídeo, por lo cual, se instaló drenaje rígido y con lumen fijado y suturado a mucosa yugal con sutura seda 3-0 (Fig. 9). Se realizó vendaje compresivo extraoral en región parotídea y geniana. La paciente fue dada de alta posterior al tratamiento ambulatorio, con indicaciones de irrigación de drenaje cada 12 horas con $10 \mathrm{ml}$ de suero fisiológico, farmacológicas con Amoxicilina $875 \mathrm{mg}$ con ácido clavulánico $125 \mathrm{mg}$ cada $12 \mathrm{~h}$, analgesia con paracetamol $1 \mathrm{~g}$ cada $8 \mathrm{~h}$ por 2 días, meloxicam $15 \mathrm{mg}$ cada $24 \mathrm{~h}$ por 5 días y colutorio de clorhexidina $0,12 \%$ para complementar higiene oral, $10 \mathrm{ml}$ cada $12 \mathrm{~h}$ por 10 días. Se realizaron controles clínicos cambiando a drenajes flexibles a las 48 h, 7 días y 15 días, retirando de manera definitiva en este último control. Evolucionó de forma asintomática, con remisión del aumento de volumen y epitelización de un nuevo coducto parotídeo, comprobado con la permeabilidad de contenido salival (Figs. 10 y 11). 

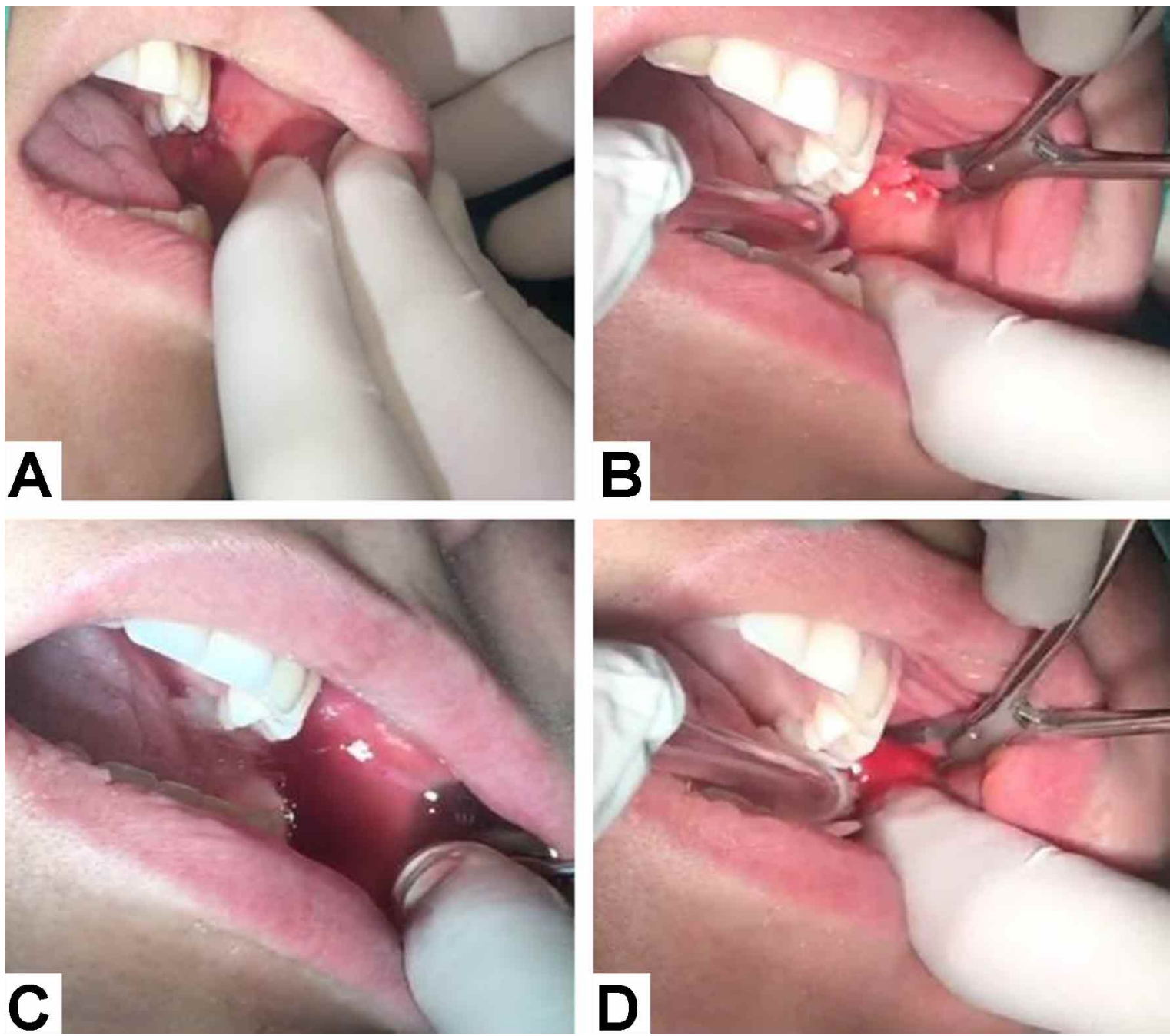

Fig. 8. Retiro de sutura, vaciamiento de exudado salival complementado y estimulada su salida con masaje infra cigomático y parotídeo. A: Comprobación de la falta de flujo salival. B: Divulsión roma de la herida. C: Salida de abundante colección salival. D: Salida de colección hemática y salival.

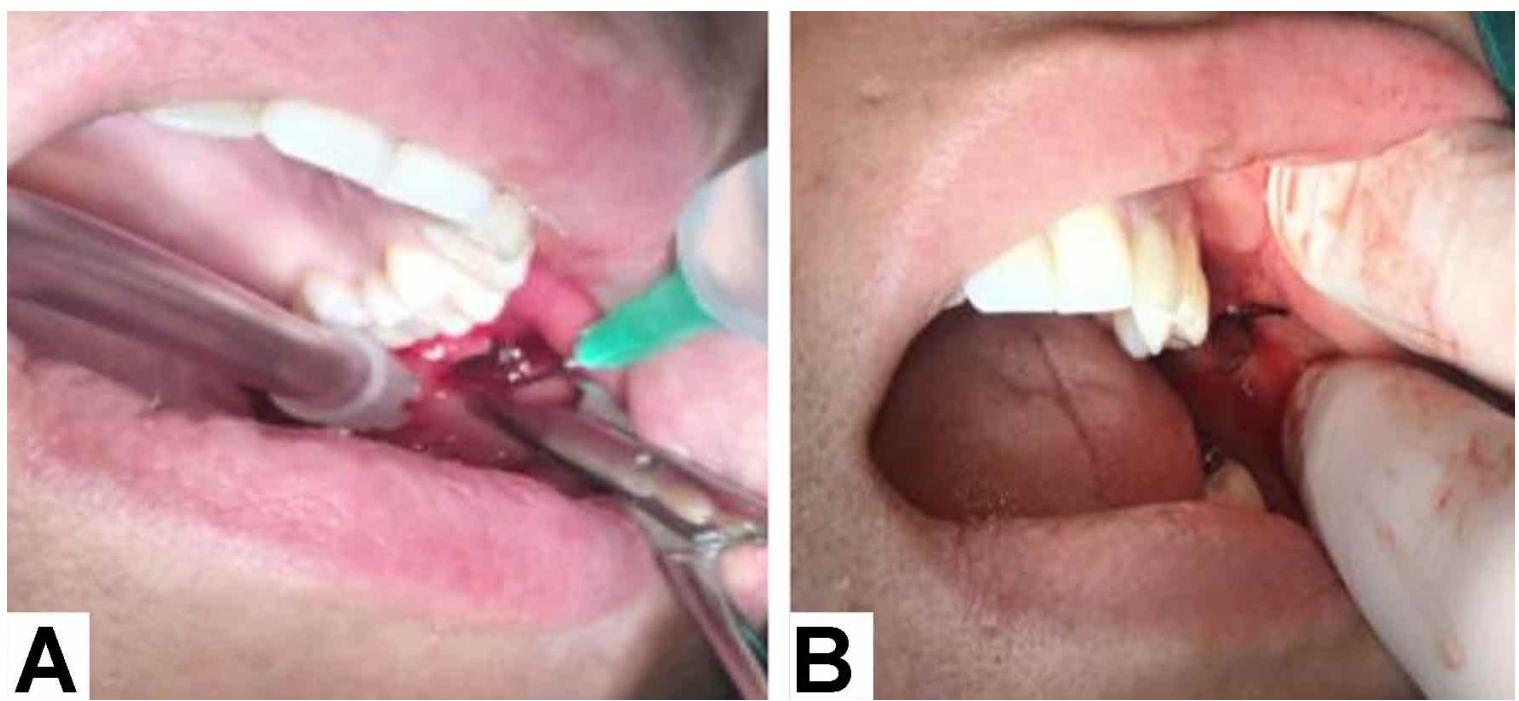

Fig. 9. A: Lavado con suero fisiológico. B: Drenaje rígido instalado. 


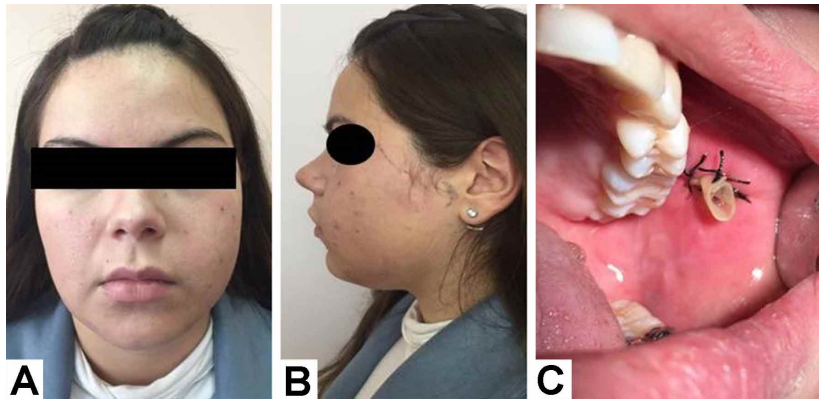

Fig. 10. A y B: Vista extraoral anterior y lateral a las 48 horas. C: Drenaje flexible instalado.

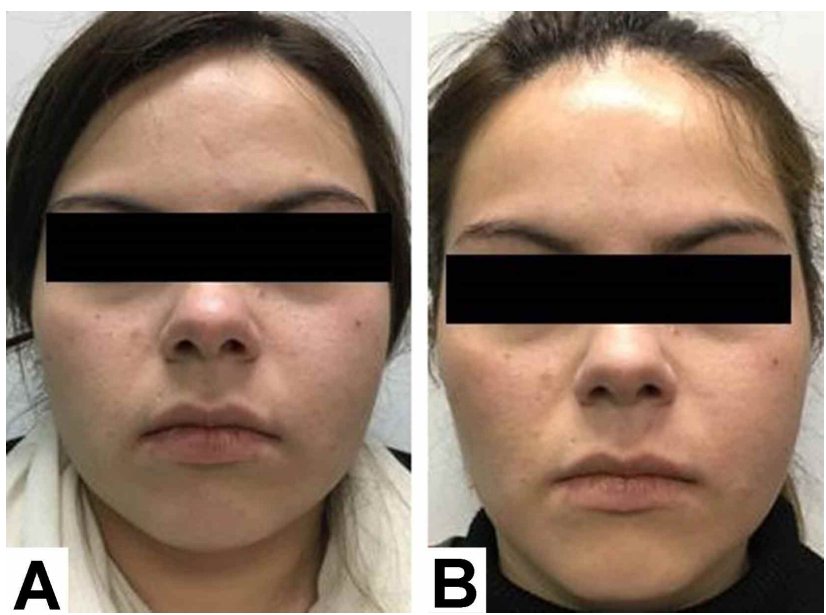

Fig. 11. A: Visión extraoral a los 7 dias. B: Visión extraoral a los 15 días.

\section{DISCUSIÓN}

En el caso presentado, la paciente consultó por aumento de volumen facial, posterior a tratamiento quirúrgico estético de CAM, asociado en primera instancia a un cuadro infeccioso. Al momento de realizar la evaluación de urgencia, se diagnosticó como sialocele consecutivo a la obstrucción traumática del conducto parotídeo. Se realizó vaciamiento, instalación de drenaje y seguimiento del paciente, remitiendo el cuadro al pasar los días. Al revisar los antecedentes del caso en retrospectiva se presume que cuando se realizó la cirugía de CAM se seccionó y posteriormente se suturó, posiblemente de manera involuntaria, el conducto parotídeo no haciéndose evidente en el momento la iatrogenia de éste que desencadena una posterior acumulación de saliva en planos profundos con resultado de aumento de volumen facial. Al revisar en la literatura actual no se encontraron casos reportados que tengan fundamentos anatómicos en describir lo sucedido.

El tratamiento en base al retiro del cuerpo adiposo de la mejilla es un tratamiento antiguo para temas funcionales (Hassani et al.; Altschiller-Mardones et al.), pero hoy en día está muy de moda para tratamiento estéticos, esto sobretodo en profesionales ligados a la odontología (Matarasso; Dias et al.; Moreira Júnior et al.). Esto ha generado un aumento de la demanda de profesionales que solicitan realizar cursos cortos o pasadas con especialistas para aprender la técnica, en ocasiones no aplicando o entregando los conocimientos anatómicos necesarios para poder intervenir la zona, lo que podría resultar en un mayor riesgo de dañar estructuras anatómicas circundantes de importancia como el conducto parotídeo, vasos faciales y elementos nerviosos. Por lo que causar la iatrogenia de estos elementos puede significar secuelas temporales y/o permanentes (Klüppel et al.).

Al revisar la literatura actual hay pocos artículos que evidencien las complicaciones desde un punto de vista anatómico (Benjamin \& Reish; Klüppel et al.). Se describe principalmente artículos en la literatura de los elementos en las zonas próximas del CAM y sus posibles implicaciones clínicas (Dubin et al., 1989; Tostevin \& Ellis; Kahn et al., 2000; Zhang et al.; Hwang et al.; Hassani et al.; Benjamin \& Reish).

Al revisar la información actual conforme al cuerpo adiposo de la mejilla se prefiere, actualmente, la descripción en lobos de Zhang et al., por sobre la descripción en cuerpo central (Tostevin \& Ellis; Kahn et al.), ya que para la descripción en lobos se utiliza los elementos de fijación e irrigación asociado a cada lobo, entregando un detalle anatómico mayor (Zhang et al.; Arce; Hassani et al.).

Los elementos importantes en la zona son varios, pero principalmente se debe considerar al nervio facial con sus ramos bucal y cigomático, al conducto parotídeo, vena facial, a los nervios y vasos infraorbitarios y bucales, arteria facial y arteria facial transversa. Estos elementos pueden distribuirse superficial o por el espesor del cuerpo adiposo de la mejilla (Zhang et al.; Hassani et al.; Benjamin \& Reish).

Los reportes indican que el conducto parotídeo puede tener tres diferentes formas de relacionarse con el CAM, esto es de vital importancia al momento de realizar el tratamiento para no dañarlo. En los estudios se aprecia que el conducto parotídeo puede estar superficial (tipo A $42 \%$ ), por superior (tipo B, $36 \%$ ); o inmerso (tipo C, $26 \%$ ) en el CAM. (Hwang et al.; Hassani et al.). Posiblemente la relación presentada en este caso sea el tipo C o inmersa, lo que implica un mayor riesgo de daño. En base a esto es importante buscar y separar cuidadosamente el conducto parotídeo del CAM, para evitar lesionarlo, durante el procedimiento.

El nervio facial, con sus ramos bucal y cigomático, tiene una estrecha relación con la prolongación bucal del CAM (Hwang et al.; Hassani et al.). Específicamente su ramo bucal, el cual es inferior al conducto parotídeo en su 
trayecto a través de la región maseterina y geniana (Matarasso). En los estudios se infiere que el cirujano, al retirar partes del CAM, igual se lleva parte de los terminales nerviosos adyacentes, el daño puede ser mayor o menor según la cantidad y ramos que se lesione (Klüppel et al.), pero no se consigna a menudo esta pérdida de inervación por los especialistas ya que los otros ramos y nervios que quedan en la zona suplen esta pérdida (Hwang et al.). Si se consigna que al dañar el ramo bucal o cigomático del nervio facial las secuelas generadas son mayores comprometiendo la función de la mímica facial (Hwang et al.).

HERNÁNDEZ, O.; ALTAMIRANO, J.; SOTO, R. \& RIVERA, A. Anatomical relationships of the adipose buccal fat pad associated with bichectomy complications. About a case. Int. J. Morphol., 39(1):123-133, 2021.

SUMMARY: The present study seeks to provide knowledge and important aspects of the anatomical structures associated with the buccal fat pad (BFP), as consequence of the report of a case of complication after bichectomy. Knowing the anatomical elements and their relationships with BFP is of vital importance, because nowadays there are too many BFP removal courses and internships, for general dentists and specialists, due to the increasing aesthetic demands of individuals seeking a finer and more stylized profile. The case presented is a female patient who consults the emergency department for an increase in facial volume after BFP extraction, which was diagnosed as sialocele, following damage to the parotid duct. When reviewing the literature and observing our anatomical preparations, the proximity of important elements is appreciated, such as the parotid duct, the zygomatic and buccal branches of the facial nerve, facial vessels such as the facial artery, facial vein and facial transverse artery. It is essential to know in detail the area in order to intervene and consider the possible anatomical variations of these elements to avoid injury during the procedure. The aimed objective of this study, is to provide accurate and concise anatomical information of important elements near or immersed in BFP, to prevent iatrogenesis.

KEY WORDS: Anatomy; Adipose Tissue / surgery; Cheek / surgery; Esthetics.

\section{REFERENCIAS BIBLIOGRÁFICAS}

Altschiller-Mardones, J.; Noguera-Pantoja, A.; Muñoz-Zavala, T.; PooleyDonoso, P. \& Solé-Ventura, P. Buccal fat pad indications as a flap and autologous graft in maxillofacial surgery. Narrative review. Int. J. Odontostomatol., 12(4):362-7, 2018.

Arce, K. Buccal fat pad in maxillary reconstruction. Atlas Oral Maxillofac. Surg. Clin. North Am., 15(1):23-32, 2007.

Benjamin, M. \& Reish, R. G. Buccal fat pad excision: proceed with caution. Plast. Reconstr. Surg. Glob. Open, 6(10):e1970, 2018.

Dias, A. C. D. S.; Teodoro, T. A. D.; Carvalho, L. P.; Dietrich, L.; Martins, L. H. B. \& Costa, M. D. M. A. Bichectomia: uma indicação cirúrgica estética e funcional na odontologia - Revisão de literatura. Rev. Psicol. Saúde Debate, 4(Suppl. 1):54, 2018.
Dubin, B.; Jackson, I. T.; Halim, A.; Triplett, W. W. \& Ferreira, M. Anatomy of the buccal fat pad and its clinical significance. Plast. Reconstr. Surg., 83(2):257-64, 1989.

Hassani, A.; Shahmirzadi, S. \& Saadat, S. Applications of the Buccal Fat Pad in Oral and Maxillofacial Surgery. Textb. Adv. Oral Maxillofac. Surg., 3, 2016. Disponible en: https://www.intechopen.com/books/atextbook-of-advanced-oral-and-maxillofacial-surgery-volume-3/ applications-of-the-buccal-fat-pad-in-oral-and-maxillofacial-surgery

Hwang, K.; Cho, H. J.; Battuvshin, D.; Chung, I. H. \& Hwang, S. H. Interrelated buccal fat pad with facial buccal branches and parotid duct. J. Craniofac. Surg., 16(4):658-60, 2005.

Kahn, J. L.; Wolfram-Gabel, R. \& Bourjat, P. Anatomy and imaging of the deep fat of the face. Clin. Anat., 13(5):373-82, 2000.

Klüppel, L.; Marcos, R. B.; Shimizu, I. A.; da Silva, M. A. D. \& da Silva, R. D. Complications associated with the bichectomy surgery. RGO Rev. Gaúch. Odontol., 66(3):278-84, 2018.

Matarasso, A. Buccal fat pad excision: aesthetic improvement of the midface. Ann. Plast. Surg., 26(5):413-8, 1991.

Moreira Júnior, R.; Gontijo, G.; Guerreiro, T. C.; de Sousa, N. L. \& Moreira, R. Bichectomia, a simple and fast surgery: case report. Rev. Odontol. Bras. Cent., 27(81):98-100, 2018.

Shoja, M. M.; Tubbs, R. S.; Loukas, M.; Shokouhi, G. \& Ardalan, M. R. Marie-François Xavier Bichat (1771-1802) and his contributions to the foundations of pathological anatomy and modern medicine. Ann. Anat., 190(5):413-20, 2008.

Stuzin, J. M.; Wagstrom, L.; Kawamoto, H. K.; Baker, T. J. \& Wolfe, S. A. The anatomy and clinical applications of the buccal fat pad. Plast. Reconstr. Surg., 85(1):29-37, 1990.

Tostevin, P. M. \& Ellis, H. The buccal pad of fat: a review. Clin. Anat., 8(6):403-6, 1995.

Zhang, H. M.; Yan, Y. P.; Qi, K. M.; Wang, J. Q. \& Liu, Z. F. Anatomical structure of the buccal fat pad and its clinical adaptations. Plast. Reconstr. Surg., 109(7):2509-18, 2002.

\section{Dirección para correspondiente: \\ Alejandro Rivera Palacios \\ Profesor Asistente \\ Facultad de Odontología \\ Universidad de los Andes \\ Monseñor Álvaro del Portillo 12455 \\ Las Condes \\ Santiago \\ CHILE}

\section{Cirujano Dentista Staff \\ Urgencia Dental y Maxilofacial \\ Unidad de EmergenciasAdultos \\ Hospital Dr. Sotero del Rio \\ Santiago \\ CHILE}

Email: arivera@miuandes.cl

Recibido : 06-08-2020

Aceptado: 17-09-2020 